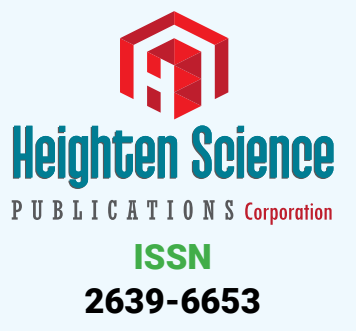

*Address for Correspondence: Farahnaz Fallahian, Pulmonology and critical care Fellow, Respiratory Diseases Department, Iran University of Medical Sciences, Tehran, Iran, Email: falahianfff@yahoo.com

Submitted: 09 January 2018

Approved: 22 January 2018

Published: 23 January 2018

Copyright: @ 2018 Fallahian F, et al. This is an open access article distributed under the Creative Commons Attribution License, which permits unrestricted use, distribution, and reproduction in any medium, provided the original work is properly cited.

Keywords: Interstitial lung disease; Lung cancer Lung fibrosis; Telomerase

Check for updates
Research Article

\section{Evaluation of Desmin, a-SMA and hTERT expression in pulmonary fibrosis and lung cancer}

Farahnaz Fallahian ${ }^{*}$, Seyed Ali Javad Moosavi², Frouzandeh

Mahjoubi ${ }^{3}$, Samira Shabani ${ }^{4}$, Pegah Babaheidarian ${ }^{5}$ and Tayebeh

Majidzadeh ${ }^{4}$

${ }^{1}$ Pulmonology and critical care Fellow, Respiratory diseases Department, Iran University of Medical Sciences, Tehran, Iran

${ }^{2}$ Professor in Pulmonology and critical care, Respiratory diseases Department, Iran University of Medical Sciences, Tehran, Iran

${ }^{3}$ Associate Professor in Medical Genetics, PhD. Clinical Genetic Department, International Institute of Genetic Engineering and Biotechnology. Tehran, Iran

${ }^{4}$ Medical Genetics, PhD, Clinical Genetic Department, International Institute of Genetic

Engineering and Biotechnology. Tehran, Iran

${ }^{5}$ Assistant Professor in Pathology, Pathology Department, Iran University of Medicall Sciences,

Tehran, Iran

\section{Abstract}

Background: Pulmonary fibrosis is a clinical problem with an enigmatic etiology with no effective therapy. Current therapies for lung fibrosis are ineffective for progression of lung fibrosis and preventing respiratory failure.

Objectives: The aim of this study is to explore the expression of Desmin, a-smooth muscle actin (a-SMA) and the telomerase subunit: human telomerase reverse transcriptase (h-TERT) in a spectrum of lung tissue samples consist of lung fibrosis, lung cancer, and healthy controls.

Materials and Methods: The expression of Desmin, a-SMA and hTERT were studied in samples of 15 pulmonary fibrosis samples, 16 samples of lung cancer and 14 healthy controls investigated. We evaluated Desmin, a-SMA as well as the expression of components of telomerase (TERT), by methods: RNA Extraction and cDNA synthesis, Real-Time quantitative PCR, Immunohistochemistry, all prepared from lung tissue paraffin blocked.

Results: a-SMA marker detected $1(8.3 \%)$ of healthy control and $11(91.7 \%)$ of lung fibrosis samples. The difference between groups was significant $(p<0.001)$. Also the difference between healthy control $1(6.7 \%)$ and lung cancer $14(93.3 \%)$ for a-SMA marker was a significant $(P<0.001)$. It was a significant difference between healthy control and lung cancer for TERT expression $(P=.005)$. TERT was not positive in any sample of neither healthy control nor lung fibrosis. For TERT, it was a significant difference between lung fibrosis and lung cancer by Fisher's Exact Test $(P=.004)$. Expression of TERT and a-SMA between small cell lung cancer $(S C L C)$ and nonsmall cell lung cancer (NSCLC) was not statistically significant $(P=.700, P=0758)$, respectively.

Conclusions: We recommend more investigation to regard a-SMA, Desmin in patients with lung fibrosis and follow them for possible cancer risk. Also, more study is needed to regard TERT as a marker in lung cancer. Assessment of these markers may have future implication to explain the same way of pathogenesis and carcinogenesis of fibrosis and cancer and for prevention or treatment.

\section{Introduction}

There is clear evidence that environmental exposures and genetic predisposition contribute to the pathogenesis of idiopathic pulmonary fibrosis (IPF). Genetic predisposition to IPF is evident from its familial aggregation and the fact that pulmonary fibrosis develops in several rare genetic disorders [1]. 
About one out of 10 patients with idiopathic pulmonary fibrosis (IPF) develop lung cancer [2]. The risk of development of lung cancer in IPF is higher for older male smokers and there is a significantly higher prevalence of lung cancer in the combined IPF and emphysema syndrome compared with fibrosis only. Furthermore, common epigenetic alterations may represent a promising target for therapeutic approaches in the near future [2]. Data on the mechanisms underlying IPF have been generated from epidemiologic investigations as well as cellular and molecular studies of human tissues. Epithelial injury, fibroblast activation and repetitive cycles of injury and abnormal repair are almost certainly key events [3].

Myofibroblasts play important roles in the pathogenesis of lung fibrosis. Transforming growth factor (TGF)-beta 1 has been widely recognized as a key fibrogenic cytokine [4]. The pathologic hallmark of idiopathic pulmonary fibrosis (IPF) and other fibrotic pulmonary diseases are fibroblastic foci, areas rich in mesenchymal cells and extracellular matrix. Fibroblast telomerase activity increases after lung injury and the subsequent decrease in telomerase activity is likely important for fibroblast to myofibroblast differentiation [5]. A subset of pulmonary fibrosis, like dyskeratosis congenita, bone marrow failure, and liver disease, represents a "telomeropathy" caused by germline mutations in telomerase and characterized by short telomere lengths. Family members within kindreds who do not inherit the TERT mutation have shorter telomere lengths than controls, demonstrating epigenetic inheritance of a shortened parental telomere length set-point [6]. Mutations in telomerase complex genes (TERT or TERC) and short telomeres are genetic risk factors for the development of familial or sporadic idiopathic pulmonary fibrosis. Up to $15 \%$ of familial cases and approximately $5 \%$ of sporadic cases carry a heterozygous mutation in one of the genes, and patients' cells retain approximately $50 \%$ of telomerase activity. Short telomeres even in the absence of telomerase mutations are a feature of most patients with idiopathic pulmonary fibrosis. Telomerase mutations also have been linked to pulmonary fibrosis and emphysema syndrome [7]. Telomere integrity is mediated by the telomerase complex, a specialized polymerase that adds sequences to the ends of chromosomes [8]. Aberrant telomerase activity has been associated with most human cancers and several premature aging diseases, such as idiopathic pulmonary fibrosis (IPF), a chronic, progressive, and fatal lung disease characterized by alveolar epithelial cell damage and fibrosis [9]. This manuscript evaluates the possible role of $\alpha$-SMA, Desmin, and TERT expression as markers of lung fibrosis process and early detection of cancer. We tested the hypothesis that expression of markers of fibrosis, and short telomeres in tissues may prone a subject for pulmonary fibrosis or cancer in a spectrum of lung tissues from healthy to fibrosis and cancer.

\section{Materials and Methods}

\section{Ethics statement}

For preparation of sample from their reserved paraffin-embedded lung tissue biopsy, informed consent was obtained from all subjects or their families and the study protocol was approved by the Ethics Committee of the University Hospital of Rasoul Akram.

\section{Patients}

We studied medical records of patients who were admitted in Rasoul Akram Hospital from 2006 to 2017, and had lung tissue biopsy samples during their workup and were diagnosed as: lung fibrosis [15], lung cancer [16], and also [14] subjects whom their samples were defined normal as control.

Totally, 45 persons were included. 20(44.4\%) were female and 25(55.6\%) were male. Their mean age was $54.42 \pm 13.236$ year. Of 15 persons of lung fibrosis group, $4(26.7 \%)$ were female and $11(73.3 \%)$ were male. Their mean age was $51.13 \pm 16.453$ 
year. Of 16 subjects of lung cancer group, 9(56.3\%) were female and 7(43.8\%) were male. Their mean age was $60.38 \pm 9.905$ year. Of 14 subjects of healthy control group, $7(50 \%)$ were female and $7(50 \%)$ were male. Their mean age was $51.14 \pm 10.982$ year. Demographic characteristic of studied population is shown in table 1. Frequency of expression of $\alpha$-SMA, Desmin, and TERT is depicted in table 2, and Figure 1. Of 16 patients of lung cancer group: 7(43.8\%) were adenocarcinoma, 7 (43.8\%) were squamous cell carcinoma (SCC), and $2(12.5 \%)$ were neuro-endocrine carcinoma. $87.5 \%$ of cancers were NSCLC, and $12.5 \%$ were SCLC. The mean age of 14 patients in non-small cell lung cancer (NSCLC) group was $9.302 \pm 60.93$ and the mean age of 2 patients of small cell lung cancer (SCLC) group was $56.50 \pm 17.678$ year. In SCLC group one patient was male and the other female. In NSCLC group of 14 patients, $6(42.9 \%)$ were male, and 8 cases (57.1\%) were female. Paraffin-embedded lung tissue blocks of these patients were sectioned and slides were stained with H\&E method. Then sections were immunostained for $\alpha$-SMA and Desmin, and all reviewed and scored by one pathologist. Alpha SMA was also scored subjectively as 0 (no staining) +1 (mild staining), +2 (moderate staining), +3 (severe staining), and +4 (very severe staining). Characteristics of $\alpha$-SMA score in studied population are depicted in table 3 .

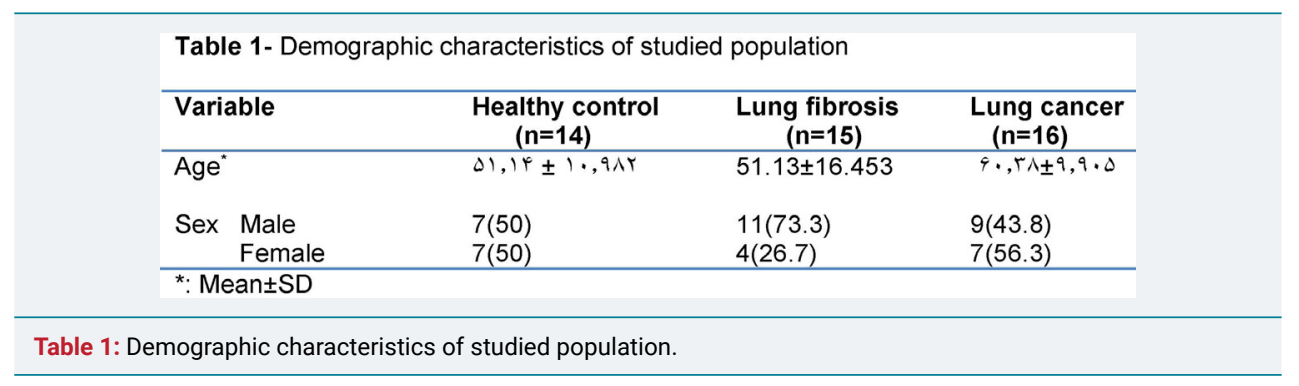

Table 2- Frequency (percent) of subjects expressing $\alpha$-SMA, Desmin, and TERT in lung tissue samples

\begin{tabular}{lccc} 
& Healthy $(\mathbf{n = 1 4})$ & Lung fibrosis $(\mathbf{n = 1 5 )}$ & Lung cancer $(\mathbf{n = 1 6})$ \\
\hline Variable & $\mathrm{n}(\%)$ & $\mathrm{n}(\%)$ & $\mathrm{n}(\%)$ \\
$\mathrm{\alpha}-\mathrm{SMA}$ & $1(7.1 \%)$ & $11(73.3 \%)$ & $14(87.5 \%)$ \\
Desmin & - & $2(13.3 \%)$ & - \\
TERT & - & - & $7(43.8 \%)$ \\
\hline
\end{tabular}

Table 2: Frequency (persent) of subjects expressing $\alpha$-SMA, Desmin, and TERT in lung tissue samples.

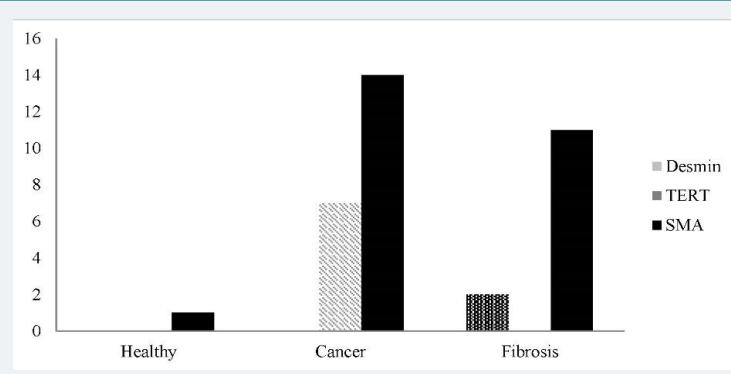

Figure 1: Distribution of frequencies of Desmin, TERT, and $\alpha$-SMA in studied population.

Table 3 - characteristics of a-SMA score in studied population

\begin{tabular}{llll} 
& Lung fibrosis & Lung cancer & Healthy control \\
\hline Valid & $\mathrm{n}(\%)$ & $\mathrm{n}(\%)$ & $\mathrm{n}(\%)$ \\
Negative & $4(26.7)$ & $2(12.5)$ & $13(92.9)$ \\
Weakly positive & $5(33.3)$ & $8(50)$ & $1(7.1)$ \\
+1 & & & \\
$+\mathrm{r}$ & $3(20)$ & $3(18.8)$ & - \\
+3 & $2(13.3)$ & $3(18.8)$ & - \\
+4 & $1(6.7)$ & - & - \\
total & $15(100)$ & $16(100)$ & $14(100)$
\end{tabular}

Table 3: Characteristics of a $\alpha$-SMA, score in studied population. 
Pathology report of some patients of fibrosis group:

1-Lung tissue with interstitial inflammation, fibrosis, emphysematous alveoli

2-Marked interstitial fibrosis

3-Iinterstitial fibrosis associated with alveolar destruction and cyst formation compatible with honeycomb lung

4-Interstitial fibrosis and inflammation with cystic alveoli (honey-combing pattern)

5-Chronic inflammation and fibrosis

6-Interstitial pneumonitis associated with reactive atypia

7-Interstitial fibrosis associated with chronic inflammation and hemorrhage

8- Diffuse interstitial fibrosis (diffuse pulmonary fibrosis)

9-Severe interstitial pneumonitis with fibrosis and destruction of alveolar structure compatible with advanced interstitial lung disease

10-Focal intraparenchymal hemorrhage, interstitial fibrosis

11- Severe acute and chronic inflammation, foreign body type giant cell reaction with fibrosis

12-interstitial pulmonary fibrosis

13-Interstitial fibrosis consistent with idiopathic pulmonary fibrosis

14-Usual interstitial pneumonia (idiopathic pulmonary fibrosis)

Pathology report of some healthy control subjects are as follow:

1-Bronchial mucosa and lung parenchyma with no significant pathologic change

2-Portion of pulmonary parenchyma with mild chronic peribronchial inflammation

3-Subpleural bullae along with congested lung parenchyma show anthracosis

4- Lung biopsy consistent with emphysematous bulla

5-Fragments of bronchial mucosa and lung parenchyma with no significant pathologic change

6- Consistent with emphysematous bulla

\section{Laboratory methods}

RNA extraction and cDNA synthesis: Total RNA extraction from paraffinembedded tissue sections of fibrosis, cancerous and normal lung was carried out by RNasy FFPE Kit (QIAgen) according to the manufacturer's instructions. RNA solutions purity and quantity was determined by measuring ultraviolet absorption ratios of 260/280 nm using the nanodrop spectrophotometer. After measuring the RNA concentration in samples we synchronized all samples, individuals whom tumorderived total RNA was inadequate for evaluation were excluded. For cDNA synthesis, $1 \mu \mathrm{g}$ of total RNA from each sample/ RNA was transcribed into single-stranded cDNA according to the manufacturer's protocol (Frementase) using oligo dT as a primer.

Real-time RT-PCR: Real-Time quantitative PCR was performed by using a Corbett 6000 Real Time Thermal cycler and Fast-Start DNA Master SYBR-Green I kit ) Roche Applied Sciences). Bactin was selected as a housekeeping gene. Primer design was carried out with oligo7 software. Primer specificity was theoretically checked by BLAST database and experimentally confirmed by the positive control amplification 


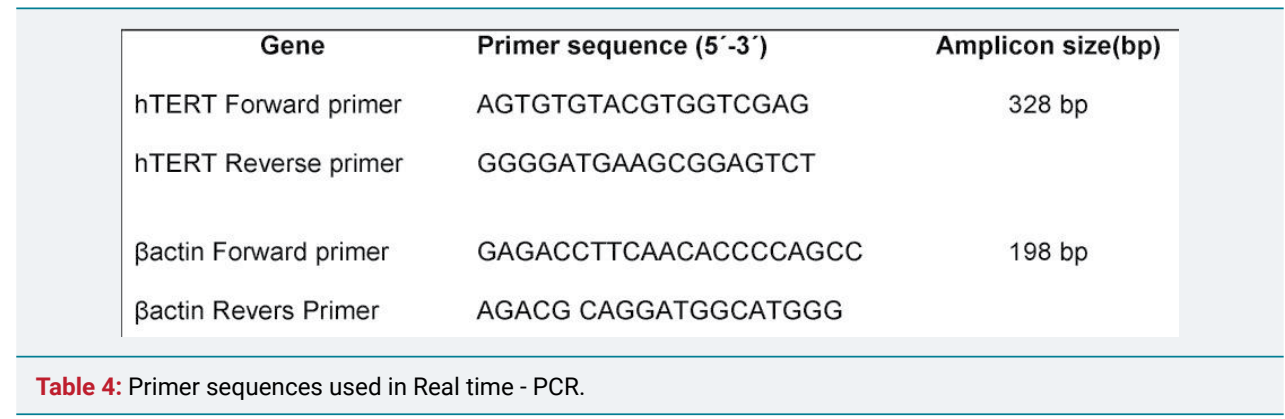

for each gene. Detailed primers information was described in table 4. The Real Time RT- PCR was performed in $10 \mu \mathrm{L}$ of solution, consisting of $2 \mu \mathrm{L}$ of Fast Start Master solution and $0.5 \mu \mathrm{M}$ of each primer, $3 \mu \mathrm{L}$ water and $4 \mu \mathrm{L}$ of cDNA as a template was placed into 0.1 vials. Thermal cycling consisted of an initial denaturation step $95^{\circ} \mathrm{C}$ for 5 min followed by an amplification program repeated for 45 cycles.

Immunohistochemistry: Immunohistochemistry were carried out according to standard protocol. $3 \mu \mathrm{m}$-thick slicing of formalin-fixed, paraffin-embedded tissue blocks were prepared and then mounted on positively charged glass slides, then the the slides were deparaffinized in xylene twice for $5 \mathrm{~min}$, and hydrated in 100\% and $96 \%$ ethanol twice for $5 \mathrm{~min}$ respectively and then slides rinsed in distilled water. Antigen retrieval was performed using Sodium Citrate Buffer (10mM Sodium Citrate, 0.05\% Tween 20, $\mathrm{pH}$ 6.0) and autoclaved for $10 \mathrm{~min}$ followed by cooling to room temperature for $15 \mathrm{~min}$. The slides were incubated in $1 \%$ hydrogen peroxide at room temperature to inhibit peroxidase activity, then slides were rinsed in distilled water and PBS buffer respectively. Slides were next incubated at room temperature for $30 \mathrm{~min}$ and were stained with the following mouse, anti-human antibodies: FLEX monoclonal mouse anti-human smooth muscle actin (clone 1A4,DAKO,A/S).Then rinsed with phosphatebuffered saline (PBS).Subsequently, the sections were exposed to secondary antibody (DAKO REAL envision detection system, Peroxidase/DAB): Envision (a peroxidaseconjugated polymer backbone, which, in addition, also carries secondary antibody molecules directed against rabbit and mouse immunoglobulins) for 30 minutes. Reaction products were detected/revealed using/by liquid DAB plus chromogenic system. Then the slides were rinse with PBS buffer for $5 \mathrm{~min}$, rinse in distilled water and Counterstain slides by immersing slides in Hematoxylin for $30 \mathrm{Sec}$. Dehydrate the tissue slides through 4 changes of alcohol (95\%, and 100\%), for 5 min each. And finally immunostaining reactions were evaluated with light microscopy by pathologist.

\section{Statistical Analysis}

The results were described as Mean $\pm S D$ and range of continuous variables. Also, frequency and percentage of categorical variables or percent Row number were reported. Fisher's Exact Test was used to determine the relationship between categorical variables. Level of significance for statistical test was 0.05 . The SPSS software version 24 was used for statistical analysis.

\section{Results}

\section{Compare of lung fibrosis group and healthy control group}

In case of sex, in healthy control group, there were 7 (63.6\%) female and 7 (38.9\%) male. In lung fibrosis group, 4 (36.4\%) subjects were female and 11 (61.1\%) were male. It was not any difference between groups for gender. $\alpha$-SMA detected in $1(8.3 \%)$ of healthy control samples and in $11(91.7 \%)$ of lung fibrosis. The statistical difference between groups by Fisher's Exact Test was significant $(\mathrm{p}<0.001)$. In none of the healthy control Desmin was positive, but it was detected in 2 samples of lung fibrosis. It was not significant difference between two groups $(\mathrm{P}=0.157)$. TERT was not expressed in any case of healthy control group, but it was positive in $7(100 \%)$ of lung cancer group. 
By Fisher's Exact Test it was a significant difference between groups $(\mathrm{P}=0.005)$.

\section{Compare of lung fibrosis and lung cancer group}

In fibrosis group 4 (30.8\%) were female and 11 (61.1\%) were female. In lung cancer 9 cases $(69.2 \%)$ were female and $7(38.9 \%)$ were male. It was no significant difference between two groups for gender.

In lung fibrosis group, $\alpha$-SMA marker was positive in $11(44.0 \%)$ and it was positive in lung cancer group in $14(56.0 \%)$. It was not a significant difference between two groups by Fisher's Exact Test $(\mathrm{P}=0.318)$.

Desmin was detected in 2(100\%) of lung fibrosis, but it was not detected in any case of lung cancer. It was not a significant difference between two groups by Fisher's Exact Test $(\mathrm{P}=0.131)$. The results of Real Time RT-PCR showed that hTERT was not expressed in any case of lung fibrosis, but it was expressed in $7(100 \%)$ of lung cancer. There was a significant difference between two groups by Fisher's Exact Test $(\mathrm{P}=0.004)$.

\section{Compare of lung cancer and healthy control group}

Of 14 healthy control group 7(50.0\%) were male and 7(43.8\%) were female. Of 16 in lung cancer: 9 (50.0\%) subjects were male and 7(56.3\%) were female. It was not any difference between groups for gender. $\alpha$-SMA was positive in $1(6.7 \%)$ subject of healthy control group and in 14(93.3\%) of lung cancer group. By Fisher's Exact Test it was a significant difference between group $(\mathrm{P}<0.001)$.

TERT was not positive in any case of healthy control group, but was positive in $7(100 \%)$ of lung cancer group. By Fisher's Exact Test it was a significant difference between groups $(\mathrm{P}=0.005)$.

\section{Compare of lung cancer types}

TERT marker was positive in 1(14.3\%) in SCLC group and in $6(85.6 \%)$ of patients of NSCLC group. With Fisher's Exact Test the difference was not significant $(\mathrm{P}=0.700)$. In SCLC group $\alpha$-SMA was positive in $2(14.3 \%)$, and in NSCLC it was positive in 12(85.7\%), ( $\mathrm{P}=0758)$. The difference was not significant. Distribution of frequencies

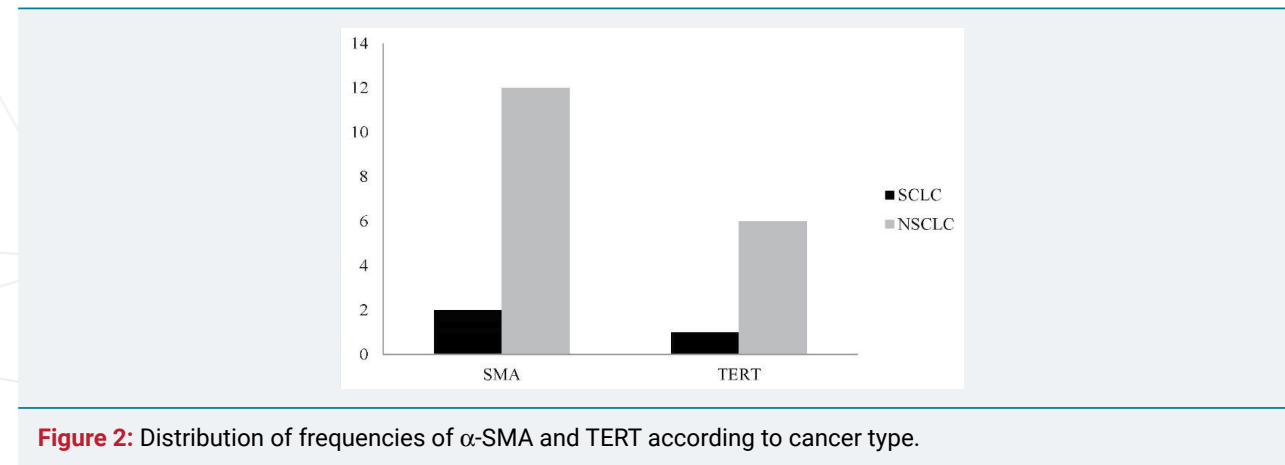

A

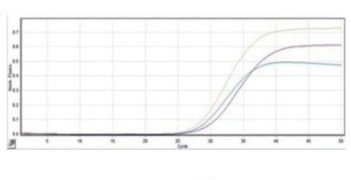

C

Figure 3: The amplification curves, melting curve and PCR products of hTERT based on Real Time RT PCR in Parafin-embedded lung cancer tissue blocks. A) Amplification curves, of hTERT, B) melting curves of hTERT and C) expression of hTERT by electrophoresis of PCR products that showed only one band of the expected size. 
of $\alpha$-SMA and TERT according to lung cancer type is depicted in figure 2. hTERT expression analysis in lung cancer by Real Time RT PCR is shown in figure 3.

\section{Discussion}

Mutations in the genes encoding telomerase (TERT and TERC) cause IPF through shortening of telomere lengths and probable exhaustion of lung stem cells. The molecular data of two seemingly unrelated biologic pathways: alveolar epithelial endoplasmic reticulum stress and telomerase dysfunction are beginning to elucidate the pathogenesis of IPF [10]. In our study, as $\alpha$-SMA was detected both in samples lung fibrosis and lung cancer, we suggest follow-up of lung fibrosis cases for possible cancer risk. However, TERT was expressed just in 7 lung cancer sample. Our study recommends regarding $\alpha$-SMA and desmin, and telomere length for evaluation and therapeutic targets for lung fibrosis, and lung cancer. However, another study [11] mentioned that the pathogenesis of pulmonary fibrosis was independent of telomere length. In a study [12], bone marrow mesenchymal stem cells (BM-MSCs) were studied in 6 Idiopathic Pulmonary Fibrosis (IPF) patients, 7 patients with Rheumatoid Arthritis associated usual interstitial pneumonia and 6 healthy controls. They demonstrated same telomere length and telomerase expression in BM-MSCs of both IPF and RA-UIP which could explain similarities in pathogenesis and prognosis [12]. The prevalence rates of lung cancer in patients with IPF (4.8\% to $48 \%$ ) are much higher than patients without IPF (2.0\% to 6.4\%). Lung cancers often occur in the peripheral lung zones where fibrotic changes are predominant. The underlying mechanisms of the development of lung cancer in patients with IPF have not been fully understood, but may include the inflammatory response, epithelial injury and/or abnormalities, aberrant fibroblast proliferation, epigenetic and genetic changes, reduced cell-tocell communication, and activation of specific signaling pathways [13]. According to a review [14], review: A recurrent global theme is that many microRNAs studied in IPF are both regulated by transforming growth factor $\beta 1$ (TGF $\beta 1$ ) and regulate TGF $\beta 1$ signaling pathway by their target genes. The changes in microRNA expression in the IPF lung and the evidence for their role in the fibrosis suggest that microRNAs should be evaluated as therapeutic targets in IPF [14]. In a study in Central Denmark, IPF was the most frequently occurring interstitial lung disease (ILD) (28\%) [15]. Telomerase dysfunction has been associated with both fibrogenesis and carcinogenesis (16). A study (16) aimed to evaluate the telomerase mRNA expression levels of both subunits (h-TERT and h-TERC) in lung tissue and bronchoalveolar lavage fluid (BALF) from patients with idiopathic pulmonary fibrosis (IPF) and non-small cell lung cancer (NSCLC), since there are indications of common pathogenetic pathways in these diseases. According to that study [16], both h-TERT and h-TERC mRNA expression is down regulated in lung tissue from patients with IPF compared with healthy controls. Telomerase is expressed in most human cancers, including lung cancers. As in many other cancer types, lung cancer cells avoid the progressive attrition of telomeres by expressing telomerase. Using a polymerase chain reaction based telomeric repeat amplification protocol assay, early studies reported finding telomerase activity in most primary lung cancer samples [17-19]. Furthermore, several studies using animal models and human non-small cell lung cancer JNSCLC) tissues have reported that TERT mRNA and TERT protein are overexpressed in lung cancer biopsies compared with normal lung tissues $[17,20,21]$.

Epithelial to mesenchymal transitions (EMT) is a complex phenomenon which is characterized by acquisition of markers of fibroblasts and myofibroblasts such as $\alpha$-smooth muscle actin ( $\alpha$-SMA) and N-cadherin and the loss of epithelial markers including E-cadherin leading to the transformation of the epithelial cell into a motile mesenchymal cell [22]. EMT is known to be an important mechanism of epithelial injury and fibrosis in several tissues [22-24]. During the EMT process, the cell junctions 
of epithelial cells are lost and the cytoskeletons are remoulded, transforming the cells into spindles from polygonal shapes and acquiring invasive attributes due to loss of polarity and adhesive property. Besides, many signal molecules in EMT are thought to be related to the genesis of carcinomas. These phenomena indicate EMT process is associated with malignant transformation of epithelial cells and the metastasis of epitheliogenic tumors $[22,25]$. In our study, TERT expression was detected in some patients with lung cancer, but there was not a significant difference between NSCLC and SCLC lung cancer for TERT expression.

\section{Conclusion}

Significant difference between expression of $\alpha$-SMA in healthy controls and lung fibrosis may $\alpha$-SMA marker be regarded as marker of fibrosis predisposition. Also significant difference between expression of $\alpha$-SMA and TERT in healthy control and lung cancer, may implement these markers for cancer susceptibility. We recommend more investigation to regard $\alpha$-SMA, Desmin in patients with fibrosis and follow them for possible cancer risk. Also more studies is needed to regard TERT as a marker in lung cancer. Assessment of these markers may have future implication to explain the same way of pathogenesis and carcinogenesis of fibrosis and cancer and for prevention or treatment.

\section{Acknowledgements}

Thanks for the statistics support of Professors Mehrdad Sheikhvatan and Atefeh Talebi.

\section{References}

1. Steele MP, Schwartz DA. Molecular mechanisms in progressive idiopathic pulmonary fibrosis. Annu Rev Med. 2013; 64: 265-276. Ref.: https://goo.gl/fczBNJ

2. Antoniou KM, Tomassetti S, Tsitoura E, Vancheri C. Idiopathic pulmonary fibrosis and lung cancer: a clinical and pathogenesis update. Curr Opin Pulm Med. 2015; 21: 626-633. Ref.: https://goo.gl/jWeuVY

3. Kottmann RM, Hogan CM, Phipps RP, Sime PJ. Determinants of initiation and progression of idiopathic pulmonary fibrosis. Respirology. 2009; 14: 917-933. Ref.: https://goo.gl/HLStPp

4. Gu L, Zhu YJ, Yang X, Guo ZJ, Xu WB, et al. Effect of TGF-beta/Smad signaling pathway on lung myofibroblast differentiation. Acta Pharmacol Sin. 2007; 28: 382-391. Ref.: https://goo.gl/XhG3A8

5. Schissel SL, Layne MD. Telomerase, myofibroblasts, and pulmonary fibrosis. Am J Respir Cell Mol Biol. 2006; 34: 520-522. Ref.: https://goo.gl/f7VWCS

6. Diaz de Leon A, Cronkhite JT, Katzenstein AL, Godwin JD, Raghu G, et al. Telomere lengths, pulmonary fibrosis and telomerase (TERT) mutations. PLoS One. 2010; 19: 5. Ref.: https://goo.gl/zJHVKp

7. Calado RT. Telomeres in lung diseases. Prog Mol Biol Transl Sci. 2014; 125: 173-183. Ref.: https://goo.gl/oY1Huh

8. Trăilă D, Mlădinescu OF, Oancea C, Tudorache V. Short telomeres in pulmonary fibrosis: from genetics to clinical significance. Pneumologia. 2015; 64: 11-13. Ref.: https://goo.gl/NuFKh5

9. Tsang AR, Wyatt HD, Ting NS, Beattie TL. hTERT mutations associated with idiopathic pulmonary fibrosis affect telomerase activity, telomere length, and cell growth by distinct mechanisms. Aging Cell. 2012; 11: 482-490. Ref.: https://goo.gl/8UVVzU

10. Garcia CK. Idiopathic pulmonary fibrosis: update on genetic discoveries. Proc Am Thorac Soc. 2011; 8: 158-162. Ref.: https://goo.gl/5HNPyX

11. Liu $T$, Ullenbruch $M$, Young Choi $Y, Y u H$, Ding $L$, et al. Telomerase and telomere length in pulmonary fibrosis. Am J Respir Cell Mol Biol. 2013; 49: 260-268. Ref.: https://goo.gl/T1cxvA

12. Antoniou KM, Margaritopoulos GA, Proklou A, Karagiannis K, Lasithiotaki I, et al. Investigation of Telomerase/Telomeres system in Bone Marrow Mesenchymal Stem Cells derived from IPF and RAUIP. J Inflamm (Lond). 2012; 9: 27. Ref.: https://goo.gl/qXSPLW

13. Li J, Yang M, Li P, Su Z, Gao P, et al. Idiopathic pulmonary fibrosis will increase the risk of lung cancer. Chin Med J (Engl). 2014; 127: 3142-3149. Ref.: https://goo.gl/zLfsgw 
14. Pandit KV, Milosevic J, Kaminski N. MicroRNAs in idiopathic pulmonary fibrosis. Transl Res. 2011; 157: 191-199. Ref.: https://goo.gl/HA6iBb

15. Hyldgaard C. A cohort study of Danish patients with interstitial lung diseases: burden, severity, treatment and survival. Dan Med J. 2015; 62: 5069. Ref.: https://goo.gl/knDj3C

16. Antoniou KM, Samara KD, Lasithiotaki I, Margaritopoulos GA, Soufla G, et al. Differential telomerase expression in idiopathic pulmonary fibrosis and non-small cell lung cancer. Oncol Rep. 2013; 30: 2617-2624. Ref.: https://goo.gl/PrJ5yx

17. Fernandez-Garcia I, Ortiz-de-Solorzano $C$, Montuenga LM. Telomeres and telomerase in lung cancer. J Thorac Oncol. 2008; 3:1085-1088. Ref.: https://goo.gl/z3F9f3

18. Hiyama K, Hiyama E, Ishioka S, Yamakido M, Inai K, et al. Telomerase activity in small-cell and nonsmall-cell lung cancers. J Natl Cancer Inst. 1995; 87: 895-902. Ref.: https://goo.gl/1aqFrm

19. Holt SE, Shay JW. Role of telomerase in cellular proliferation and cancer. J Cell Physiol. 1999; 180: 10-18. Ref.: https://goo.gl/94G1jS

20. Lantuejoul S, Salon C, Soria JC, Brambilla E. Telomerase expression in lung preneoplasia and neoplasia. Int J Cancer. 2007; 120: 1835-1841. Ref.: https://goo.gl/XFajYs

21. Blanco D, Vicent S, Fraga MF, Fernandez-Garcia I, Freire J, et al. Molecular analysis of a multistep lung cancer model induced by chronic inflammation reveals epigenetic regulation of p16 and activation of the DNA damage response pathway. Neoplasia. 2007; 9: 840-852. Ref.: https://goo.gl/kG65Q7

22. Yan Wang, Yiting Liu, Qinghua Zhang, Rong Gao, Ke Wang. IPF and lung cancer: homologous but different endings, the progress in the correlation research. Int J Clin Exp Med. 2017; 10:4319-4329. Ref.: https://goo.gl/dm2gNS

23. Liu Y. Epithelial to mesenchymal transition in renal fibrogenesis: pathologic significance, molecular mechanism, and therapeutic interven-tion. J Am Soc Nephrol. 2004; 15: 1-12. Ref.: https://goo.gl/hYCT68

24. Pozharskaya V, Torres-Gonzalez E, Rojas M, Gal A, Amin M, et al. Twist: a regulator of epithelialmesenchymal transition in lung fibrosis. PLoS One. 2009; 4: 7559. Ref.: https://goo.gl/1mPvbu

25. Thiery JP. Epithelial-mesenchymal transitions in tumour progression. Nat Rev Cancer. 2002; 2: 442454. Ref.: https://goo.gl/eNhjdo 